\title{
Properties of galaxies around the most massive SMBHs
}

\author{
Yuji Shirasaki $^{1,2}$, Yutaka Komiya ${ }^{2}$, Masatoshi Ohishi ${ }^{1,2}$ \\ and Yoshihiko Mizumoto ${ }^{1,2}$ \\ ${ }^{1}$ National Astronomical Observatory of Japan, \\ 2-21-1 Osawa, Mitaka, Tokyo 181-8588, Japan \\ ${ }^{2}$ The Graduate University for Advanced Studies (SOKENDAI), \\ 2-21-1 Osawa, Mitaka, Tokyo 181-8588, Japan \\ ${ }^{3}$ Research center for the early universe, The University of Tokyo, \\ Hongo 7-3-1, Bunkyo-ku, Tokyo 113-0033, Japan
}

\begin{abstract}
We present result of the clustering analysis performed between AGNs and galaxies. AGN samples with redshift 0.1-1.0 were extracted from AGN properties catalogs which contain virial mass estimates of SMBHs. Galaxy samples were extracted from SDSS DR8 catalog and UKIDSS DR9 LAS catalog. The catalogs of SDSS and UKIDSS were merged and used to estimate the IR-opt color and IR magnitude in the rest frame by SED fitting. As we had no redshift information on the galaxy samples, stacking method was applied. We investigated the BH mass dependence of cross correlation length, red galaxy fraction at their environment, and luminosity function of galaxies. We found that the cross correlation length increase above $M_{\mathrm{BH}} \geqslant 10^{8.2} M_{\odot}$, and red galaxies dominate the environment of AGNs with $M_{\mathrm{BH}} \geqslant 10^{9} M_{\odot}$. This result indicates that the most massive SMBHs are mainly fueled by accretion of hot halo gas.
\end{abstract}

Keywords. galaxies: active, large-scale structure of universe, quasars: general

\section{Summary}

The open questions are the nature of the fueling of SMBHs and triggering mechanisms of AGNs. To distinguish the most effective modes at each epoch in the evolution of $\mathrm{SMBH}$, we investigated the properties (clustering, color, luminosity function) of galaxies around AGNs as a function of their SMBH mass. The AGN samples are derived from two AGN properties catalogs by Shen et al. (2011) and Greene et al. (2007). The galaxies samples are derived from UKIDSS DR9 LAS catalog and SDSS DR8 photometric catalog. In total 8059 AGN samples are analyzed in this work. Cross correlation lengths are calculated for each BH mass group. Significant increase of cross correlation length is seen at $M_{\mathrm{BH}} \geqslant 10^{8.2} M_{\odot}$ (Komiya et al. 2013). Color distributions for galaxies around AGNs are also obtained and they are fitted with a two component (blue and red galaxies) model. We found that the environment of AGNs with BH mass $\geqslant 10^{9.0} M_{\odot}$ is enriched with red galaxies (Shirasaki et al. 2015). These result indicate that the feeding of SMBH can be mostly dominated by a hot halo mode.

\section{References}

Greene, J. E. \& Ho, L. C., 2007, ApJ, 667, 131

Komiya, Y. et al. 2013, ApJ, 775, 43

Shen, Y. et al. 2011, ApJS, 194, 45

Shirasaki, Y. et al. 2015, arXive:1503.08893 